\title{
Lower bound on the neutralino mass from new data on CMB and implications for relic neutralinos
}

\author{
A. Bottino, ${ }^{*}$ F. Donato ${ }^{\dagger}$ N. Fornengo, ${ }^{\ddagger}$ and S. Scopel ${ }^{\S}$ \\ Dipartimento di Fisica Teorica, Università di Torino, Istituto Nazionale di Fisica Nucleare, Sezione di Torino, via P. Giuria 1, I-10125 \\ Torino, Italy \\ (Received 14 April 2003; published 8 August 2003)
}

\begin{abstract}
In the framework of an effective minimal supersymmetric extension of the standard model without gauginomass unification at a grand unification scale, we set a lower bound on the neutralino mass based on the new WMAP data on $\Omega_{\mathrm{CDM}}\left(R\right.$-parity conservation is assumed). Our lower bound $m_{\chi} \geq 6 \mathrm{GeV}$ leaves much room for relic neutralinos significantly lighter than those commonly considered ( $m_{\chi} \geq 50 \mathrm{GeV}$ ). We prove that these light neutralinos may produce measurable effects in weakly interacting massive particle direct detection experiments of low energy threshold and large exposure.

DOI: 10.1103/PhysRevD.68.043506

PACS number(s): 95.35.+d, 11.30.Pb, 12.60.Jv, 95.30.Cq
\end{abstract}

\section{INTRODUCTION}

In a previous paper [1], we called attention to relic neutralinos of light masses, i.e., with a mass $m_{\chi} \lesssim 45 \mathrm{GeV}$. Actually, most analyses on cosmological neutralinos employ a lower bound $m_{\chi} \geq 50 \mathrm{GeV}$, i.e., a bound which rests on the assumption that gaugino masses are unified at the grand unification (GUT) scale $M_{\text {GUT }} \sim 10^{16} \mathrm{GeV}$. However, this unification assumption might not be justified, as already discussed some time ago [2,3]. Furthermore, recent analyses of string models (see, for instance, Ref. [4]) indicate that the initial scale for the running of the supersymmetry (SUSY) parameters by renormalization group equations may be much lower than the standard GUT scale, with the implication that at the electroweak (EW) scale the gaugino masses may be quite different from what is expected in a standard supergravity (SUGRA) scheme with GUT-unification assumptions. Thus, for instance, the $\mathrm{U}(1)$ and $\mathrm{SU}(2)$ gaugino masses $M_{1}$ and $M_{2}$ may not be related by the standard formula $M_{1} \simeq \frac{1}{2} M_{2}$ at the EW scale.

It is then quite natural to discuss supersymmetric models where $M_{1}$ and $M_{2}$ are considered as independent parameters. Previous papers where schemes of this type are considered include the ones in Refs. [2,3,5-18]. In Ref. [1] we evaluated the neutralino relic abundance $\Omega_{\chi} h^{2}$ and the neutralinonucleon scalar cross section $\sigma_{\text {scalar }}^{\text {(nuclen) }}$ in an effective minimal supersymmetric extension of the standard model (EMSSM) where the gaugino-mass unification at GUT scale is not assumed. The analysis was performed in a scenario where the ratio $R \equiv M_{1} / M_{2}$ is smaller than the GUT-unification value $R_{\mathrm{GUT}} \simeq 0.5$, thus allowing the neutralino mass $m_{\chi}$ to be lighter than the commonly used lower bound of about 50 $\mathrm{GeV}$. We showed that, in the derivation of the lower limit on $m_{\chi}$, the upper bound on the relic abundance for cold dark matter (CDM), $\Omega_{\mathrm{CDM}} h^{2}$, plays a crucial role. A lower limit

\footnotetext{
*Electronic address: bottino@to.infn.it

†Electronic address: donato@to.infn.it

*Electronic address: fornengo@to.infn.it

${ }^{\S}$ Electronic address: scopel@to.infn.it; http://www.to.infn.it/ astropart
}

of $m_{\chi} \gtrsim 5 \mathrm{GeV}$ was derived in Ref. [1] by employing the upper bound $\Omega_{\mathrm{CDM}} h^{2} \lesssim 0.3$. It was also shown how this upper bound on $\Omega_{\mathrm{CDM}} h^{2}$ is instrumental in providing sizeable values for the neutralino-nucleon scalar cross section at small $m_{\chi}$.

New data on the cosmic microwave background (CMB) [19-21], also used in combination with other cosmological observations, are progressively narrowing down the ranges of the relic abundances for matter $\left(\Omega_{m} h^{2}\right)$ and for some of its constituents: neutrinos $\left(\Omega_{\nu} h^{2}\right)$ and baryons $\left(\Omega_{b} h^{2}\right)$. As a consequence also the range of $\Omega_{\mathrm{CDM}} h^{2}$ is reaching a unprecedented level of accuracy. The importance of an improvement in the determination of $\Omega_{\mathrm{CDM}} h^{2}$ for any cold relic particle is twofold. The upper bound $\left(\Omega_{\mathrm{CDM}} h^{2}\right)_{\max }$ obviously establishes a strict upper limit for any specific cold species. On the other side, the lower bound $\left(\Omega_{\mathrm{CDM}} h^{2}\right)_{\min }$ fixes the value of the average abundance below which the halo density of a specific cold constituent has to be rescaled as compared to the total CDM halo density. For the determination of the rescaling factor $\xi \equiv \rho_{\chi} / \rho_{0}$ (where $\rho_{\chi}$ and $\rho_{0}$ are the local neutralino density and the total local dark matter density, respectively), we use the standard rescaling recipe $\xi=\min \left[1, \Omega_{\chi} h^{2} /\left(\Omega_{\mathrm{CDM}} h^{2}\right)_{\min }\right]$. In Ref. [19] ranges for $\Omega_{m} h^{2}$ and $\Omega_{b} h^{2}$ are derived by employing CMB data of Refs. [19-21], 2dFGRS measurements [22], and Lyman $\alpha$ forest data [23]. From the values quoted in Ref. [19] for $\Omega_{m} h^{2}$ and $\Omega_{b} h^{2}$ and allowing for a $2-\sigma$ range in $\Omega_{\mathrm{CDM}} h^{2}$, one obtains: $\left(\Omega_{\mathrm{CDM}} h^{2}\right)_{\min }=0.095$ and $\left(\Omega_{\mathrm{CDM}} h^{2}\right)_{\max }$ $=0.131$. These are the values we will use here. However, one should cautiously still be open to some possible changes in these values as new cosmological observational data will accumulate in the future.

In the present paper we analyze the supersymmetric scenario of Ref. [1] in light of these new determinations of $\Omega_{\mathrm{CDM}} h^{2}$. We first derive approximate analytic expressions which directly relate the lower bound on $m_{\chi}$ to the upper limit on $\Omega_{\mathrm{CDM}} h^{2}$ ( $R$-parity conservation is assumed). This requires the evaluation of $\Omega_{\chi} h^{2}$, which is in itself a very complicated function of the supersymmetric parameters. In order to extract the leading analytic terms at small values of $m_{\chi}$, we are guided by a detailed numerical analysis of the supersymmetric parameter space. This will allow us to 
present analytic expressions for the lower bounds on $m_{\chi}$ which display the link of these bounds to the relevant particle-physics and cosmological constraints, such as the lower bounds on sfermions and Higgs-bosons masses and the upper limit on $\Omega_{\mathrm{CDM}} h^{2}$. Furthermore, on the basis of these results, we show that light neutralinos, with masses $m_{\chi}$ $\$ 45 \mathrm{GeV}$, may actually be probed by weakly interacting massive particle (WIMP) direct detection experiments with high sensitivities and low energy thresholds.

\section{LOWER BOUND ON $M_{X}$}

The supersymmetric scheme adopted here is the same as the one described in Ref. [1]: an effective MSSM scheme (EMSSM) at the electroweak scale, with the following independent parameters: $M_{2}, \mu, \tan \beta, m_{A}, m_{\tilde{q}}, m_{\tilde{l}}, A$, and $R$ $\equiv M_{1} / M_{2}$. Notations are as follows: $\mu$ is the Higgs mixing mass parameter, $\tan \beta$ the ratio of the two Higgs vacuum expectation values (VEV's), $m_{A}$ the mass of the $C P$-odd neutral Higgs boson, $m_{\tilde{q}}$ is a squark soft-mass common to all squarks, $m_{\tilde{l}}$ is a slepton soft-mass common to all sleptons, $A$ is a common dimensionless trilinear parameter for the third family, $A_{\tilde{b}}=A_{\tilde{t}} \equiv A m_{\tilde{q}}$, and $A_{\tilde{\tau}} \equiv A m_{\tilde{l}}$ (the trilinear parameters for the other families being set equal to zero).

The neutralino is defined as the lowest-mass linear superposition of $B$-ino $\widetilde{B}, W$-ino $\widetilde{W}^{(3)}$, and of the two Higgsino states $\widetilde{H}_{1}^{0}, \widetilde{H}_{2}^{0}$ :

$$
\chi \equiv a_{1} \widetilde{B}+a_{2} \widetilde{W}^{(3)}+a_{3} \widetilde{H}_{1}^{0}+a_{4} \widetilde{H}_{2}^{0} .
$$

Since we are here interested in light neutralinos, we consider values of $R$ lower than its GUT value: $R_{\mathrm{GUT}} \simeq 0.5$; for definiteness, we take $R$ in the range $0.01-0.5$.

We first outline the procedure for deriving analytical bounds on $m_{\chi}$ from the cosmological upper limit on $\Omega_{\mathrm{CDM}} h^{2}$. As mentioned in the previous section, the identification of the leading analytic contributions is guided by numerical analysis. This is based on a scanning of the supersymmetric parameter space, with the following ranges of the MSSM parameters: $1 \leqslant \tan \beta \leqslant 50,100 \mathrm{GeV}$ $\leqslant|\mu|, M_{2}, m_{\tilde{q}}, m_{\tilde{l}} \leqslant 1000 \mathrm{GeV}, \quad \operatorname{sign}(\mu)=-1,1, \quad 90 \mathrm{GeV}$ $\leqslant m_{A} \leqslant 1000 \mathrm{GeV}$, and $-3 \leqslant A \leqslant 3$. The following experimental constraints are imposed: accelerators data on supersymmetric and Higgs boson searches (CERN $e^{+} e^{-}$collider LEP2 [24] and Collider Detector CDF at Fermilab [25]); measurements of the $b \rightarrow s+\gamma$ decay [26]; and measurements of the muon anomalous magnetic moment $a_{\mu} \equiv\left(g_{\mu}\right.$ -2 ) $/ 2$ [27] (the range $-160 \leqslant \Delta a_{\mu} \cdot 10^{11} \leqslant 680$ is used here for the deviation of the current experimental world average from the theoretical evaluation within the standard model; for the derivation see Ref. [1]).

The neutralino relic abundance is given by

$$
\Omega_{\chi} h^{2}=\frac{x_{f}}{g_{\star}\left(x_{f}\right)^{1 / 2}} \frac{3.3 \times 10^{-38} \mathrm{~cm}^{2}}{\left\langle\widetilde{\sigma_{\mathrm{ann}}} v\right\rangle},
$$

where $\left\langle\widetilde{\sigma_{\mathrm{ann}}} v\right\rangle \equiv x_{f}\left\langle\sigma_{\mathrm{ann}} v\right\rangle_{\text {int }},\left\langle\sigma_{\mathrm{ann}} v\right\rangle_{\text {int }}$ being the integral from the present temperature up to the freeze-out tempera- ture $T_{f}$ of the thermally averaged product of the annihilation cross section times the relative velocity of a pair of neutralinos, $x_{f}$ is defined as $x_{f} \equiv m_{\chi} / T_{f}$ and $g_{\star}\left(x_{f}\right)$ denotes the relativistic degrees of freedom of the thermodynamic bath at $x_{f}$. For $\left\langle\widetilde{\sigma_{\mathrm{ann}}} v\right\rangle$ we will use the standard expansion in $\mathrm{S}$ and $\mathrm{P}$ waves: $\left\langle\sigma_{\mathrm{ann}} v\right\rangle \simeq \tilde{a}+\tilde{b}\left(2 x_{f}\right)$.

A lower bound on $m_{\chi}$ is now derived from Eq. (2), by requiring that

$$
\Omega_{\chi} h^{2} \leqslant\left(\Omega_{\mathrm{CDM}} h^{2}\right)_{\max }
$$

We first work out approximate analytic expressions for $\left\langle\widetilde{\sigma_{\text {ann }}} v\right\rangle$ in the regime of small $m_{\chi}\left(m_{\chi} \lesssim 45 \mathrm{GeV}\right)$. By diagonalizing the usual neutralino mass matrix in the approximation $M_{1} \ll M_{2}, \mu$, it turns out that light neutralinos have a dominant $B$-ino component; a deviation from a pure $B$-ino composition is mainly due to a mixture with $\widetilde{H}_{1}^{0}$, i.e., $\left|a_{1}\right|$ $\gg\left|a_{3}\right| \gg\left|a_{1}\right|,\left|a_{4}\right|$. For the ratio $\left|a_{3}\right| /\left|a_{1}\right|$ one finds

$$
\frac{\left|a_{3}\right|}{\left|a_{1}\right|} \simeq \sin \theta_{W} \sin \beta \frac{m_{Z}}{\mu} \lesssim 0.42 \sin \beta,
$$

where in the last step we have taken into account the experimental lower bound $\mu \gtrsim 100 \mathrm{GeV}$.

The dominant terms in $\left\langle\widetilde{\sigma_{\mathrm{ann}}} v\right\rangle_{\text {int }}$ are the contributions due to Higgs-exchange in the $s$ channel and sfermion-exchange in the $t, u$ channels of the annihilation process $\chi+\chi \rightarrow \bar{f}+f$ (interference terms are neglected). We retain only the leading terms in each contribution. Thus, for the Higgs-exchange contribution, dominated by the $\mathrm{S}$-wave annihilation into down-type fermions, we have, for any final state $\bar{f}-f$,

$$
\begin{aligned}
\left\langle\widetilde{\sigma_{\mathrm{ann}}} v\right\rangle_{f}^{\mathrm{Higgs}} \simeq & \tilde{a}_{f}^{\mathrm{Higgs}} \simeq \frac{2 \pi \alpha_{e . m .}^{2} c_{f}}{\sin ^{2} \theta_{W} \cos ^{2} \theta_{W}} a_{1}^{2} a_{3}^{2} \tan ^{2} \beta\left(1+\epsilon_{f}\right)^{2} \\
& \times \frac{\bar{m}_{f}^{2}}{m_{W}^{2}} \frac{m_{\chi}^{2}\left[1-m_{f}^{2} / m_{\chi}^{2}\right]^{1 / 2}}{\left[\left(2 m_{\chi}\right)^{2}-m_{A}^{2}\right]^{2}}
\end{aligned}
$$

where $c_{f}$ is a standard color factor $\left(c_{f}=3\right.$ for quarks, $c_{f}$ $=1$ for leptons), $\bar{m}_{f}$ is the fermion running mass evaluated at the energy scale $2 m_{\chi}$, and $m_{f}$ is the fermion pole mass. $\epsilon_{f}$ is a quantity which enters in the relationship between the down-type fermion running mass and the corresponding Yukawa coupling (see Ref. [28] and references quoted therein); in the following evaluations, $\epsilon_{f}$ is negligible, except for the bottom quark, where $\epsilon_{b} \simeq 0.2$. One easily verifies that when $m_{\chi}<m_{b},\left\langle\widetilde{\sigma_{\text {ann }}} v\right\rangle_{f}^{\text {Higgs }}$ entails a relic abundance exceeding the cosmological bound.

Notice that $\left\langle\widetilde{\sigma_{\mathrm{ann}}} v\right\rangle$ turns out to be an increasing function of $m_{\chi}$. Then, to obtain a conservative lower bound on $m_{\chi}$ from the condition of Eq. (3), we have first to evaluate an $\left(\Omega_{\chi} h^{2}\right)_{\min }$ which is obtained from Eq. (2), by replacing $\left\langle\widetilde{\sigma_{\text {ann }}} v\right\rangle$ with its maximal value $\left(\left\langle\widetilde{\sigma_{\text {ann }}} v\right\rangle\right)_{\max }$, at fixed $m_{\chi}$. In the case of Higgs-exchange contributions, this $\left(\left\langle\widetilde{\sigma_{\mathrm{ann}} v}\right\rangle\right)_{\max }$ is obtained by inserting into Eq. (5) the maximal value of the product $a_{1}^{2} a_{3}^{2} \tan ^{2} \beta$. Taking into account Eq. (4) and that, for 


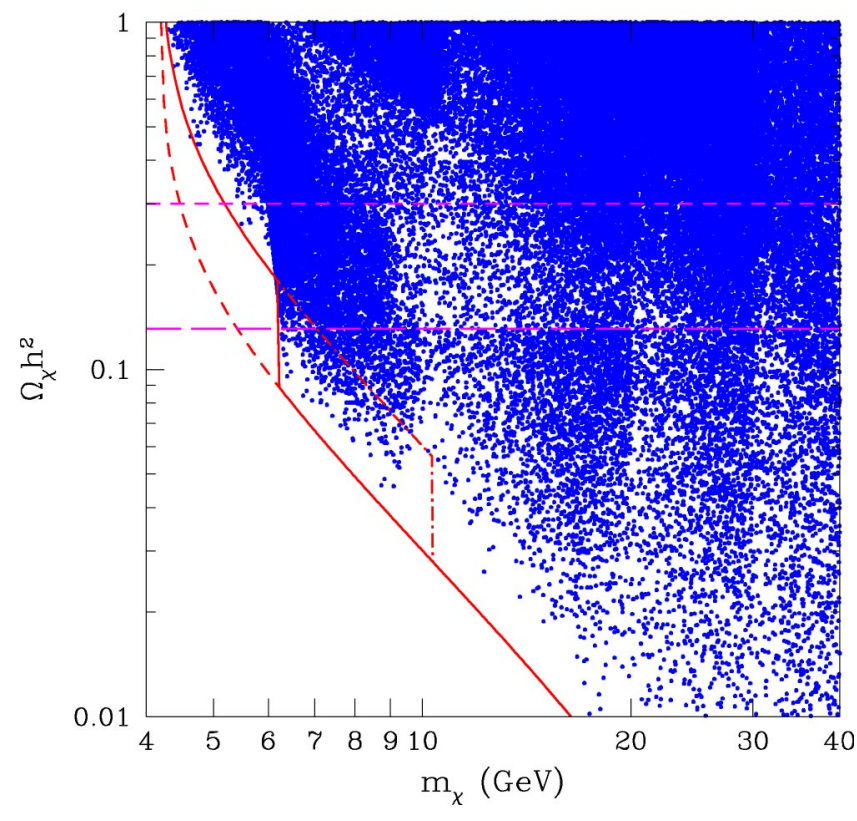

FIG. 1. Neutralino relic abundance $\Omega_{\chi} h^{2}$ as a function of the mass $m_{\chi}$. The solid curve denotes $\left(\Omega_{\chi} h^{2}\right)_{\text {min }}^{\text {Higgs }}$ given by Eq. (6) for $T_{\mathrm{QCD}}=300 \mathrm{MeV}$. Dashed and dot-dashed curves refer to the representative values $T_{\mathrm{QCD}}=100 \mathrm{MeV}, T_{\mathrm{QCD}}=500 \mathrm{MeV}$, respectively. The two horizontal lines denote two representative values of $\Omega_{\mathrm{CDM}} h^{2}: \Omega_{\mathrm{CDM}} h^{2}=0.3$ (short-dashed line) and $\Omega_{\mathrm{CDM}} h^{2}=0.131$ (long-dashed line). The scatter plot is obtained by a full scanning of the supersymmetric parameter space.

$m_{A} \simeq 90 \mathrm{GeV}$, the upper bound of $\tan \beta$ is 45 [25], we obtain $\left(a_{1}^{2} a_{3}^{2} \tan ^{2} \beta\right)_{\max } \simeq 2.6 \times 10^{2}$, and in turn

$$
\begin{aligned}
\left(\Omega_{\chi} h^{2}\right)_{\mathrm{min}}^{\mathrm{Higgs}} \equiv & \frac{1.5 \times 10^{-10}}{\mathrm{GeV}^{2}} \frac{x_{f}}{g_{\star}\left(x_{f}\right)^{1 / 2}} \frac{m_{W}^{2}}{m_{\chi}^{2}}\left[\left(2 m_{\chi}\right)^{2}-m_{A}^{2}\right]^{2} \\
& \times\left(\sum_{f} \bar{m}_{f}^{2}\left(1+\epsilon_{f}\right)^{2} c_{f}\left[1-m_{f}^{2} / m_{\chi}^{2}\right]^{1 / 2}\right)^{-1} \\
\simeq & \frac{5 \times 10^{-11}}{\mathrm{GeV}^{2}} \frac{x_{f}}{g_{\star}\left(x_{f}\right)^{1 / 2}} \frac{m_{W}^{2}}{m_{b}^{2}} \\
& \times \frac{1}{\left(1+\epsilon_{b}\right)^{2}} \frac{\left[\left(2 m_{\chi}\right)^{2}-m_{A}^{2}\right]^{2}}{m_{\chi}^{2}\left[1-m_{b}^{2} / m_{\chi}^{2}\right]^{1 / 2}} .
\end{aligned}
$$

In the last step of this equation we have retained only the dominant contribution due to the $b-\bar{b}$ final state. As far as the value of $g_{\star}\left(x_{f}\right)^{1 / 2}$ is concerned, we notice that for these light neutralinos $x_{f} \simeq 21$ to 22 , so that neutralinos with masses $m_{\chi} \simeq 6$ to $7 \mathrm{GeV}$ have a freeze-out temperature $T_{f}$ $\sim T_{\mathrm{QCD}}$, where $T_{\mathrm{QCD}}$ is the hadron-quark transition temperature of order $300 \mathrm{MeV}$. For definiteness, we describe here the hadron-quark transition by a step function: if $T_{\mathrm{QCD}}$ is set equal to $300 \mathrm{MeV}$, then for $m_{\chi} \lesssim 6 \mathrm{GeV}$ one has $g_{\star}\left(x_{f}\right)^{1 / 2}$ $\simeq 4$, while for heavier neutralinos $g_{\star}\left(x_{f}\right)^{1 / 2} \simeq 8$ to 9 .

The quantity $\left(\Omega_{\chi} h^{2}\right)_{\mathrm{min}}^{\mathrm{Higgs}}$ as given by Eq. (6) is plotted in Fig. 1 as a function of $m_{\chi}$, for the value $m_{A}=90 \mathrm{GeV}$ (cur- rent experimental lower bound). The solid curve refers to the case $T_{\mathrm{QCD}}=300 \mathrm{MeV}$; dashed and dot-dashed curves denote two different representative values of $T_{\mathrm{QCD}}: T_{\mathrm{QCD}}$ $=100 \mathrm{MeV}$ and $T_{\mathrm{QCD}}=500 \mathrm{MeV}$, respectively. The two horizontal lines denote two representative values for the upper bound on $\Omega_{\mathrm{CDM}} h^{2}:\left(\Omega_{\mathrm{CDM}} h^{2}\right)_{\max }=0.3$ (short-dashed line) and $\left(\Omega_{\mathrm{CDM}} h^{2}\right)_{\max }=0.131$ (long-dashed line). Figure 1 displays how a lower bound on $m_{\chi}$ is derived from an upper limit on $\Omega_{\mathrm{CDM}} h^{2}$. In particular, using $T_{\mathrm{QCD}}=300 \mathrm{MeV}$, one obtains from $\left(\Omega_{\mathrm{CDM}} h^{2}\right)_{\max }=0.3$ the bound $m_{\chi} \gtrsim 5.2 \mathrm{GeV}$, a value which increases to $m_{\chi} \geq 6.2 \mathrm{GeV}$, when the new value $\left(\Omega_{\mathrm{CDM}} h^{2}\right)_{\max }=0.131$ is employed. Also evident is the variation of the lower bound on $m_{\chi}$, when the value of $T_{\mathrm{QCD}}$ is changed. Finally, to support the validity of the analytical approximations employed to derive Eq. (6), in Fig. 1 we also display the scatter plot of $\Omega_{\chi} h^{2}$, when a numerical scanning of the supersymmetric parameter space is performed.

We recall that the bound on $m_{\chi}: m_{\chi} \geq 6.2 \mathrm{GeV}$ was derived using $m_{A}=90 \mathrm{GeV}$ (which is the present experimental lower bound on $m_{A}$ ). From our previous formulas one obtains that this bound on $m_{\chi}$ simply scales with $m_{A}$ as follows:

$$
m_{\chi}\left[1-m_{b}^{2} / m_{\chi}^{2}\right]^{1 / 4} \gtrsim 5.3 \mathrm{GeV}\left(\frac{m_{A}}{90 \mathrm{GeV}}\right)^{2}
$$

For the sfermion-exchange contribution, $\left\langle\sigma_{\mathrm{ann}} v\right\rangle_{f}^{\text {sfermion }}$ $\simeq \widetilde{a}_{f}^{\text {sfermion }}+\widetilde{b}\left(2 x_{f}\right)_{f}^{\text {sfermion }}$, both $\mathrm{S}$-wave and P-wave contributions have to be taken into account. In the regime we consider here, the leading terms due to the down-type fermions are

$$
\begin{aligned}
\widetilde{a}_{f}^{\text {sfermion }} \simeq & \frac{\pi \alpha_{e . m .}^{2}}{8 \cos ^{4} \theta_{W}} a_{1}^{4} c_{f} \frac{m_{f}^{2}\left[1-m_{f}^{2} / m_{\chi}^{2}\right]^{1 / 2}}{m_{\tilde{f}, 1}^{4}\left(2-m_{\tilde{f}, 1}^{2} / m_{\tilde{f}}^{2}\right)^{2}} \\
& \times\left[\left(Y_{f, L}^{2}+Y_{f, R}^{2}\right)+2 Y_{f, L} Y_{1, R} \frac{m_{\chi}}{m_{f}}\left(1-\frac{m_{\tilde{f}, 1}^{2}}{m_{\tilde{f}}^{2}}\right)\right]^{2},
\end{aligned}
$$

$$
\begin{aligned}
\tilde{b}_{f}^{\text {sfermion }} \simeq & \frac{\pi \alpha_{e . m .}^{2}}{4 \cos ^{4} \theta_{W}} a_{1}^{4} c_{f} \frac{m_{\chi}^{2}\left[1-m_{f}^{2} / m_{\chi}^{2}\right]^{1 / 2}}{m_{\tilde{f}, 1}^{4}\left(2-m_{\tilde{f}, 1}^{2} / m_{\tilde{f}}^{2}\right)^{2}} \\
& \times\left[2\left(Y_{f, L}^{4}+Y_{f, R}^{4}\right)+3 Y_{f, L}^{2} Y_{f, R}^{2}\left(1-\frac{m_{\tilde{f}, 1}^{2}}{m_{\tilde{f}}^{2}}\right)^{2}\right] .
\end{aligned}
$$

Notations are as follows: $Y_{f, L}$ and $Y_{f, R}$ are the weak hypercharges for left and right couplings, respectively. $m_{\tilde{f}}$ denotes either $m_{\tilde{l}}$ or $m_{\tilde{q}}$ depending on the nature of the fermion, $m_{\tilde{f}, 1}$ is the smallest mass eigenvalue for the sfermion $\widetilde{f}$, once the mass matrix in the weak-interaction basis $\widetilde{f}_{L}, \widetilde{f}_{R}$ is diagonalized. A maximal mixing between the $\widetilde{f}_{L}, \widetilde{f}_{R}$ fields has been used in deriving Eqs. (8) and (9), since these equations are meant to provide a conservative lower bound on $m_{\chi}$. Notice 


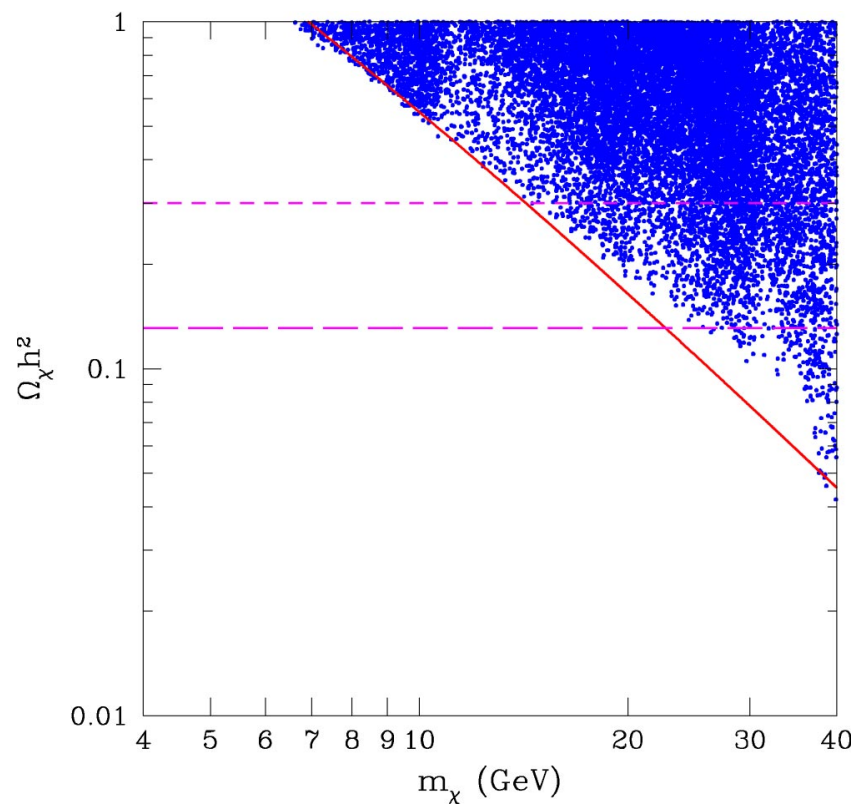

FIG. 2. Neutralino relic abundance $\Omega_{\chi} h^{2}$ as a function of the mass $m_{\chi}$. The solid curve denotes $\left(\Omega_{\chi} h^{2}\right)_{\text {min }}^{\text {sfermion }}$ derived from Eq. (2) when $\left\langle\widetilde{\sigma_{\text {ann }}} v\right\rangle$ is given by the maximal value of $\left\langle\sigma_{\text {ann }}^{\sim} v\right\rangle^{\text {sfermion }}$ [see Eq. (10)]. $T_{\mathrm{QCD}}$ is set equal to $300 \mathrm{MeV}$. The two horizontal lines denote two representative values of $\Omega_{\mathrm{CDM}} h^{2}: \Omega_{\mathrm{CDM}} h^{2}=0.3$ (short-dashed line) and $\Omega_{\mathrm{CDM}} h^{2}=0.131$ (long-dashed line). The scatter plot is obtained by a full scanning of the supersymmetric parameter space with $m_{A}>300 \mathrm{GeV}$.

that for $l=e, \mu$, the $\widetilde{f}_{L}-\widetilde{f}_{R}$ mixing is negligible; this case is simply recovered from Eqs. (8) and (9) by setting $m_{\tilde{f}, 1}$ $=m_{\tilde{f}}$.

With the aid of numerical evaluations, it is found that the leading contributions to $\left\langle\widetilde{\sigma_{\text {ann }}} v\right\rangle^{\text {sfermion }} \equiv \Sigma_{f}\left\langle\widetilde{\sigma_{\text {ann }}} v\right\rangle_{f}^{\text {sfermion }}$ are provided by the term due to the $\tau$ lepton: $\left\langle\sigma_{\text {ann }} v\right\rangle^{\text {sfermion }}$ $\simeq\left\langle\widetilde{\sigma_{\text {ann }}} v\right\rangle_{\tau}^{\text {sfermion }}$. This is in turn maximized by

$$
\begin{aligned}
\left(\left\langle\widetilde{\sigma_{\mathrm{ann}} v}\right\rangle\right)_{\text {max }}^{\text {sfermion }} \simeq & \frac{\pi \alpha_{e . m .}^{2}}{8 \cos ^{4} \theta_{W}} \frac{m_{\chi}^{2}\left[1-m_{\tau}^{2} / m_{\chi}^{2}\right]^{1 / 2}}{m_{\tilde{\tau}}^{4}} \\
& \times\left[\left(2+\frac{5}{2} \frac{m_{\tau}}{m_{\chi}}\right)^{2}+\frac{23}{2 x_{f}}\right] .
\end{aligned}
$$

We denote by $\left(\Omega_{\chi} h^{2}\right)_{\text {min }}^{\text {sfermion }}$ the value of $\Omega_{\chi} h^{2}$ derived from Eq. (2) when $\left\langle\widetilde{\sigma_{\text {ann }}} v\right\rangle$ is replaced by $\left(\left\langle\widetilde{\sigma_{\text {ann }}} v\right\rangle\right)_{\text {max }}^{\text {sfermion }}$ of Eq. (10). The quantity $\left(\Omega_{\chi} h^{2}\right)_{\text {min }}^{\text {sfermion }}$ is plotted in Fig. 2 for the value $m_{\tilde{\tau}}=87 \mathrm{GeV}$ (current experimental lower bound); also the scatter plot for the quantity $\Omega_{\chi} h^{2}$ is displayed, for $m_{A}>300 \mathrm{GeV}$. From Fig. 2 one finds for $m_{\chi}$ a lower bound $m_{\chi} \gtrsim 14 \mathrm{GeV}$ for $\left(\Omega_{\mathrm{CDM}} h^{2}\right)_{\max }=0.3$, and $m_{\chi}$ $\gtrsim 22 \mathrm{GeV}$ for $\left(\Omega_{\mathrm{CDM}} h^{2}\right)_{\max }=0.131$. The scaling of this last bound with the stau mass is approximately given by

$$
m_{\chi}\left[1-m_{\tau}^{2} / m_{\chi}^{2}\right]^{1 / 4} \geq 22 \mathrm{GeV}\left(\frac{m_{\tau}}{90 \mathrm{GeV}}\right)^{2}
$$

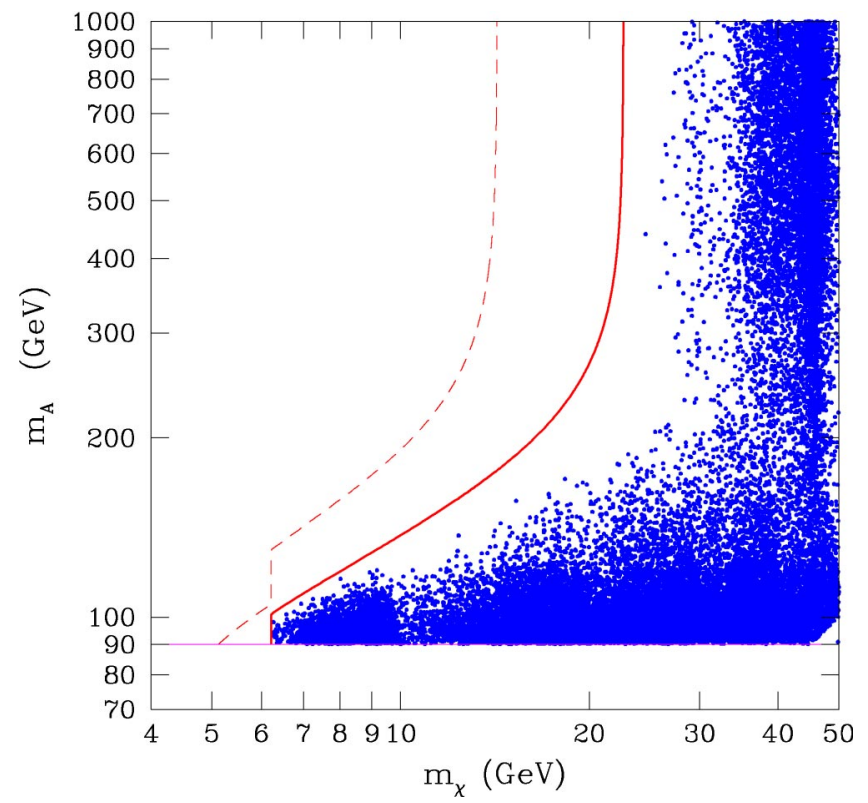

FIG. 3. Dashed and solid curves give the variation of the lower bound on $m_{\chi}$ as a function of $m_{A}$ for $\left(\Omega_{\mathrm{CDM}} h^{2}\right)_{\max }=0.3$ and for $\left(\Omega_{\mathrm{CDM}} h^{2}\right)_{\max }=0.131$, respectively. For each value of $\left(\Omega_{\mathrm{CDM}} h^{2}\right)_{\max }$ the region on the left of the relevant curve is forbidden. The two lines are derived from the analytical expressions obtained in the text for the contributions to $\left\langle\widetilde{\sigma_{\mathrm{ann}}} v\right\rangle$ due to Higgs-exchange and sfermion-exchange. Also displayed is the scatter plot of a full numerical scanning.

In general, one has conservatively to retain as a lower bound to $m_{\chi}$ the smaller of the two lower limits given separately in Eq. (7) and in Eq. (11). From these equations one finds that the lower bound of Eq. (7) is less stringent than the one of Eq. (11) as long as $m_{A} \lesssim 2 m_{\tau}$. Due to the present experimental bounds on $m_{A}$ and $m_{\tau}$ the lower absolute bound is the one derived from Eq. (7), i.e., $m_{\chi} \geq 6.2 \mathrm{GeV}$. We parenthetically note that the lower limits $m_{\chi} \gtrsim(15-18) \mathrm{GeV}$ found in Refs. $[17,18]$ are due to the assumption that $m_{A}$ is very large $\left(m_{A} \sim 1 \mathrm{TeV}\right)$.

A plot which shows the transition between the lower bounds established by considering Higgs-exchange contributions and sfermion-exchange contributions is reported in Fig. 3. As was derived by use of the approximate expressions in Eqs. (7)-(11), the transition point is at $m_{A} \sim 200-300 \mathrm{GeV}$.

\section{DETECTABILITY OF LIGHT NEUTRALINOS BY WIMP DIRECT MEASUREMENTS}

Now we wish to show that a part of the neutralino population of small $m_{\chi}$ is indeed explorable with experiments of WIMP direct detection with current sensitivities. Let us start by giving an approximate relation between the scalar neutralino-nucleon cross section and the neutralino relic abundance, valid when $\sigma_{\text {scalar }}^{\text {(nucleon) }}$ is dominated by $h$-exchange in the $t$-channel and $\sigma_{\text {ann }}$ by $A$-exchange in the annihilation $s$-channel. From the approximate formulas given in Ref. [1] for $\sigma_{\text {scalar }}^{\text {(nucleon) }}$ and $\Omega_{\chi} h^{2}$ we obtain 


$$
\begin{aligned}
\left(\Omega_{\chi} h^{2}\right) \sigma_{\text {scalar }}^{\text {(nucleon })} \simeq & 1.4 \times 10^{-40} \mathrm{~cm}^{2} \mathrm{~T}\left(\frac{m_{s}\langle N|\bar{s}| N\rangle}{200 \mathrm{MeV}}\right)^{2} \\
& \times \frac{\mathrm{GeV}^{2}}{m_{\chi}^{2}\left[1-m_{b}^{2} / m_{\chi}^{2}\right]^{1 / 2}}\left(\frac{m_{A}}{m_{h}}\right)^{4}
\end{aligned}
$$

where $m_{h}$ is the mass of the lightest $C P$-even neutral Higgs boson and $T$ is given by

$$
T=\frac{\left(a_{3} \sin \alpha+a_{4} \cos \alpha\right)^{2}}{\left(a_{4} \cos \beta-a_{3} \sin \beta\right)^{2}} \frac{\left[\sin \alpha+\epsilon_{s} \cos (\alpha-\beta) \sin \beta\right]^{2}}{\sin ^{2} \beta\left(1+\epsilon_{b}\right)^{2}} .
$$

In Eqs. (12) and (13) $\langle N|\bar{s} s| N\rangle$ is the $s$-quark density matrix element over the nucleonic state and $\alpha$ is the angle which rotates the Higgs fields $H_{1}^{(0)}$ and $H_{2}^{(0)}$ into the mass eigenstates $h$ and $H$. The approximations employed in deriving Eqs. (12) and (13) imply $\alpha \sim \pi / 2, m_{h} \sim m_{A} \sim 100 \mathrm{GeV}$, so that $T$ is of order one.

Thus for neutralino configurations with $m_{\chi} \lesssim 20 \mathrm{GeV}$, $\sigma_{\text {scalar }}^{\text {(nucleon) }}$ turns out to be bounded by

$$
\sigma_{\text {scalar }}^{\text {(nucleon) }} \geq \frac{10^{-40} \mathrm{~cm}^{2}}{\left(\Omega_{\mathrm{CDM}} h^{2}\right)_{\max }} \frac{\mathrm{GeV}^{2}}{m_{\chi}^{2}\left[1-m_{b}^{2} / m_{\chi}^{2}\right]^{1 / 2}}
$$

In deriving Eq. (14), we have set $m_{s}\langle N|\bar{s} s| N\rangle=200 \mathrm{MeV}$ [29].

The results of complete numerical evaluations of the quantity $\xi \sigma_{\text {scalar }}^{\text {(nucleon) }}$, where all relevant diagrams for the neutralino-nucleon scalar cross section and for the relic abundance are taken into account, are displayed in Fig. 4. The peculiar funnel in the scatter plot for $m_{\chi} \lesssim 20 \mathrm{GeV}$ is due to the bound of Eq. (14).

As was pointed out in Ref. [1], the present upper limits to $\xi \sigma_{\text {scalar }}^{\text {(nucleon }}$ provided by WIMP direct detection experiments [30-33] do not significantly constrain the supersymmetric configurations for the light neutralinos displayed in Fig. 4. Instead, these configurations may be relevant for experiments of direct detection with a low energy threshold and a large exposure. An experiment with these features is the DAMA/NaI experiment, whose results, after 4-years running with a total exposure of $\simeq 58000 \mathrm{~kg}$ day, show an annualmodulation effect at a $4 \sigma$ C.L. which does not appear to be related to any possible source of systematics [34]. The analysis carried out by the DAMA Collaboration to explain their modulation effect in terms of a WIMP with coherent elastic scattering was targeted to a neutralino in the frame of a usual supersymmetric scheme with gaugino-mass unification at GUT, with a consequent lower bound on the neutralino mass above $30 \mathrm{GeV}$. This interpretation was proved to be consistent with supersymmetric models with gaugino-unification at GUT [35].

Here we have considered a different supersymmetric scheme which includes significantly lower neutralino masses; thus in order to establish the possible relevance of our low-mass neutralinos for an annual-modulation effect, we have to proceed to an extension of previous analyses. To

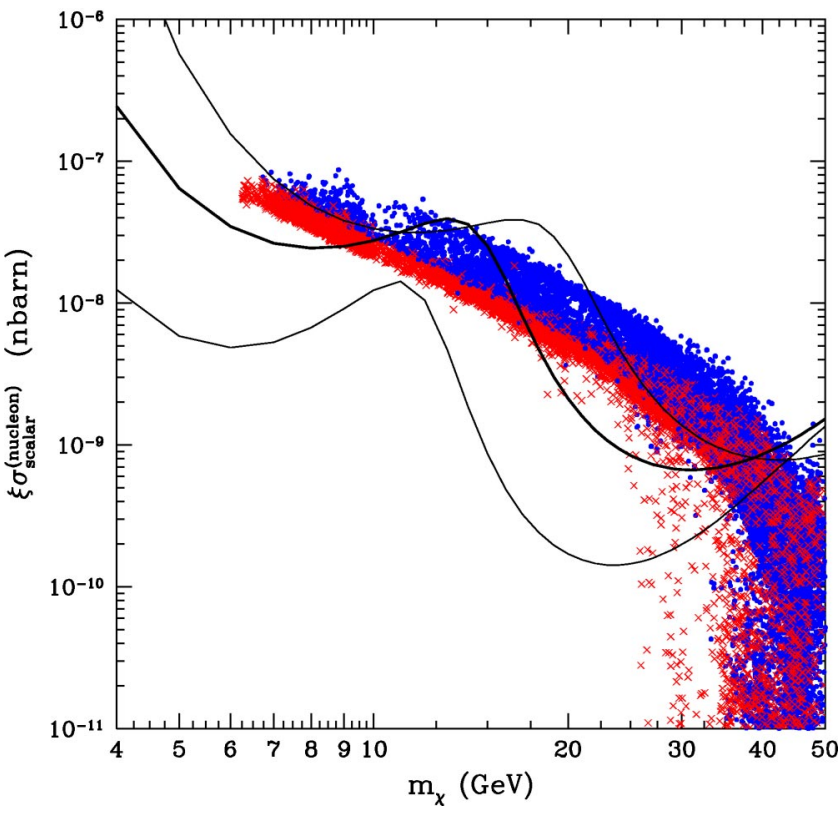

FIG. 4. Scatter plot of $\xi \sigma_{\text {scalar }}^{\text {(nucleon) }}$ vs $m_{\chi}$. Crosses (red) and dots (blue) denote neutralino configurations with $\Omega_{\chi} h^{2}>\left(\Omega_{\mathrm{CDM}} h^{2}\right)_{\min }$ and $\Omega_{\chi} h^{2}<\left(\Omega_{\mathrm{CDM}} h^{2}\right)_{\min }$, respectively. The curves give the sensitivity line, $\left(\xi \sigma_{\text {scalar }}^{\text {(nucleon })}\right)_{\min }$ vs $m_{\chi}$, for a NaI detector, whose features are discussed in the text. The intermediate curve refers to an isothermal DF with $v_{0}=220 \mathrm{~km} \mathrm{~s}^{-1}$ and $\rho_{0}=0.3 \mathrm{GeV} \mathrm{cm}^{-3}$. The upper curve refers to a spherical Evans' power-law DF (denoted as A3 in Ref. [38]) with $v_{0}=170 \mathrm{~km} \mathrm{~s}^{-1}$ and $\rho_{0}=0.17 \mathrm{GeV} \mathrm{cm}^{-3}$; the lower curve refers to an axially symmetric Evans' logarithmic DF with maximal flatness (denoted as $\mathrm{C} 2$ in Ref. [38]) with $v_{0}$ $=270 \mathrm{~km} \mathrm{~s}^{-1}$ and $\rho_{0}=1.7 \mathrm{GeV} \mathrm{cm}^{-3}$.

put our arguments into a quantitative basis, we evaluate $\left(\xi \sigma_{\text {scalar }}^{(\text {nucleon })}\right)_{\min }$, defined as the minimal value of $\xi \sigma_{\text {scalar }}^{\text {(nucleon) }}$ which may produce an annual-modulation effect at $n$ standard deviations in a detector with a given exposure $S$, and for a given velocity distribution function $f(\vec{v})$ for relic neutralinos in our galaxy, i.e.,

$$
\left(\xi \sigma_{\text {scalar }}^{(\text {nucleon })}\right)_{\min }=\frac{n^{2}}{S} \frac{I}{(\Delta I)^{2}} .
$$

In Eq. (15) $I$ is defined as the ratio of the expected direct detection rate, integrated over an energy range $\left(E_{1}, E_{2}\right)$, to the neutralino-nucleon scalar cross section $\xi \sigma_{\text {scalar }}^{\text {(nucleon) }}$ :

$$
I=\frac{1}{\xi \sigma_{\text {scalar }}^{(\text {nucleon })}} \int_{E_{1}}^{E_{2}} \frac{d R(E)}{d E} d E
$$

For a monoatomic material of nuclear mass number $A$, one has

$$
I=N_{T} \rho_{0} \frac{m_{N}}{2 m_{\chi}^{3}}\left(1+\frac{m_{\chi}}{m_{p}}\right)^{2} A^{2} \int_{E_{1}}^{E_{2}} d E F^{2}(E) \int_{v_{\min }(E)}^{\infty} d \vec{v} \frac{f(\vec{v})}{|\vec{v}|}
$$


where $N_{T}$ is the number of the target nuclei per unit of mass, $m_{N}$ is the nuclear mass, $F(E)$ the nuclear form factor, and $v_{\min }$ is the minimal value of the neutralino velocity to produce an event above the detection threshold. Generalization of Eq. (17) to nonmonoatomic materials is straightforward. In Eq. (15) $\Delta I$ is defined as $\Delta I \equiv[I$ (June) $-I$ (December) $] / 2$.

We emphasize that, by definition, the condition $\xi \sigma_{\text {scalar }}^{\text {(nucleon) }} \geqslant\left(\xi \sigma_{\text {scalar }}^{\text {(nucleon) }}\right)_{\min }$ only establishes a minimal requirement for a neutralino of a given mass $m_{\chi}$ to be detected in a given experiment. It is obvious that the real detectability of the signal depends on the actual amount of the experimental background. For example, an equal amount of signal and background would double the value of $\left(\xi \sigma_{\text {scalar }}^{\text {(nucleon) }}\right)_{\min }$ as given in Eq. (15). Estimates of backgrounds are omitted here.

To establish whether our population of light neutralinos may be relevant for the effect measured by the DAMA experiment, we have evaluated $\left(\xi \sigma_{\text {scalar }}^{(\text {nucleon })}\right)_{\min }$ as a function of $m_{\chi}$; the energy range of integration employed here is $E_{1}$ $=2 \mathrm{keV}, E_{2}=3 \mathrm{keV}$, and $n$ is set equal to 4 . The nuclear form factors for $\mathrm{Na}$ and $I$ are modelled in the Helm form [36], with the values of parameters given in Ref. [37]. In Fig. 4 we give the curves of $\left(\xi \sigma_{\text {scalar }}^{\text {(nucleon })}\right)_{\min }$ versus $m_{\chi}$ for a sample of different galactic distribution functions (DF), taken among those analyzed in Ref. [38]. The intermediate curve refers to an isothermal DF with $v_{0}=220 \mathrm{~km} \mathrm{~s}^{-1}$ and $\rho_{0}=0.3 \mathrm{GeV} \mathrm{cm}^{-3}\left(v_{0}\right.$ is the local rotational velocity). The upper curve refers to a spherical Evans' power-law DF (denoted as A3 in Ref. [38]) with $v_{0}=170 \mathrm{~km} \mathrm{~s}^{-1}$ and $\rho_{0}$ $=0.17 \mathrm{GeV} \mathrm{cm}^{-3}$; the lower curve refers to an axially symmetric Evans' logarithmic DF with maximal flatness (denoted as C2 in Ref. [38]) with $v_{0}=270 \mathrm{~km} \mathrm{~s}^{-1}$ and $\rho_{0}$ $=1.7 \mathrm{GeV} \mathrm{cm}^{-3}$.

From the results displayed in Fig. 4 one sees that indeed a part of our population of relic neutralinos of small $m_{\chi}$ may be relevant for the annual-modulation effect discussed above, especially in case of DF's which entail higher velocities and/or higher densities as compared to the standard isothermal distribution. This is a new interesting option which adds to the other, still valid, possibility which we discussed in the papers of Ref. [35], on relic neutralinos with masses above $50 \mathrm{GeV}$.

\section{CONCLUSIONS}

We have considered phenomenological properties of relic neutralinos in a range of low masses $\left(m_{\chi} \lesssim 45 \mathrm{GeV}\right)$, which is allowed in a MSSM model without gaugino-mass unification at a grand unification scale. We have numerically evaluated the relevant relic abundance and the neutralino-nucleon cross section, and discussed our numerical results in terms of analytic formulas, which display the connections among cosmological properties and particle-physics parameters.

Using the latest determinations of $\Omega_{\mathrm{CDM}} h^{2}$ and assuming $R$-parity conservation, we have shown that in a MSSM model without gaugino-mass unification the lower bound on the neutralino mass is $m_{\chi} \gtrsim 6 \mathrm{GeV}$. We have shown analytically how this bound is linked to the cosmological upper bound on $\Omega_{\mathrm{CDM}} h^{2}$ and to the lower limits on masses of Higgs bosons and sfermions.

The implications of light relic neutralinos with masses $\lesssim 45 \mathrm{GeV}$ for WIMP direct searches have been analyzed. It is found that these neutralinos are actually relevant for WIMP direct detection experiments of low energy threshold and large exposure. The present results extend to small masses our previous analyses about the effects of relic neutralinos with masses above $50 \mathrm{GeV}$ [35].

\section{ACKNOWLEDGMENTS}

We acknowledge Research Grants funded jointly by Ministero dell'Istruzione, dell'Università e della Ricerca (MIUR), by Università di Torino, and by Istituto Nazionale di Fisica Nucleare within the Astroparticle Physics Project.
[1] A. Bottino, N. Fornengo, and S. Scopel, Phys. Rev. D 67, 063519 (2003).

[2] J. Ellis, K. Enqvist, D.V. Nanopoulos, and K. Tamvakis, Phys. Lett. 155B, 381 (1985).

[3] M. Drees, Phys. Lett. 158B, 409 (1985); Phys. Rev. D 33, 1468 (1986).

[4] E. Gabrielli, S. Khalil, C. Munõz, and E. Torrente-Lujan, Phys. Rev. D 63, 025008 (2001).

[5] M. Drees and X. Tata, Phys. Rev. D 43, 2971 (1991).

[6] K. Griest and L. Roszkowski, Phys. Rev. D 46, 3309 (1992).

[7] S. Mizuta, D. Ng, and M. Yamaguchi, Phys. Lett. B 300, 96 (1993)

[8] A. Gabutti, M. Olechowski, S. Cooper, S. Pokorski, and L. Stodolsky, Astropart. Phys. 6, 1 (1996).

[9] G. Bélanger, F. Boudjema, F. Donato, R. Godbole, and S. Rosier-Lees, Nucl. Phys. B581, 3 (2000).

[10] A. Corsetti and P. Nath, Phys. Rev. D 64, 125010 (2001).

[11] R. Arnowitt, B. Dutta, and Y. Santoso, hep-ph/001244.
[12] D.G. Cerdeño, S. Khalil, and C. Muñoz, hep-ph/0105180.

[13] V.A. Bednyakov and H.V. Klapdor-Kleingrothaus, Phys. Rev. D 63, 095005 (2001).

[14] H. Baer, C. Balazs, A. Belyaev, R. Dermisek, A. Mafi, and A. Mustafayev, J. High Energy Phys. 05, 061 (2002).

[15] V. Bertin, E. Nezri, and J. Orloff, J. High Energy Phys. 02, 046 (2003).

[16] A. Birkedal-Hansen and B.D. Nelson, Phys. Rev. D 67, 095006 (2003); Phys. Rev. D 64, 015008 (2001).

[17] G. Bélanger, F. Boudjema, A. Pukhov, and S. Rosier-Lees, hep-ph/0212227.

[18] D. Hooper and T. Plehn, Phys. Lett. B 562, 18 (2003).

[19] D.N. Spergel et al. (WMAP), astro-ph/0302209.

[20] T.J. Pearson et al. (CBI), astro-ph/0205388.

[21] C.L. Kuo et al. (ACBAR), astro-ph/0212289.

[22] W.J. Percival et al., Mon. Not. R. Astron. Soc. 327, 1297 (2001).

[23] R.A.C. Croft et al., Astrophys. J. 581, 20 (2002); N.Y. Gnedin 
and A.J.S. Hamilton, Mon. Not. R. Astron. Soc. 334, 107 (2002).

[24] ALEPH Collaboration, A. Colaleo, talk at SUSY'01, 2001, Dubna, Russia; DELPHI Collaboration, J. Abdallah et al., DELPHI 2001-085 CONF 513, 2001.

[25] T. Affolder et al., Phys. Rev. Lett. 86, 4472 (2001).

[26] CLEO Collaboration, S. Ahmed et al., CONF 99/10, hep-ex/9908022; ALEPH Collaboration, R. Barate et al., Phys. Lett. B 429, 169 (1998); Belle Collaboration, K. Abe et al., ibid. 511, 151 (2001).

[27] Muon $(g-2)$ Collaboration, G.W. Bennett et al., Phys. Rev. Lett. 89, 101804 (2002); 89, 129903(E) (2002).

[28] A. Bottino, N. Fornengo, and S. Scopel, Nucl. Phys. B608, 461 (2001).

[29] A. Bottino, F. Donato, N. Fornengo, and S. Scopel, Astropart. Phys. 13, 215 (2000); 18, 205 (2002).

[30] For a summary on WIMP direct detection experiments, see, for instance, A. Morales, Nucl. Phys. B (Proc. Suppl.) 110, 39 (2002).

[31] DAMA/NaI Collaboration, R. Bernabei et al., Phys. Lett. B 389, 757 (1996).

[32] CDMS Collaboration, D. Abrams et al., Phys. Rev. D 66, 122003 (2002).

[33] A. Benoit et al., Phys. Lett. B 545, 43 (2002).

[34] DAMA/NaI Collaboration, R. Bernabei et al., Phys. Lett. B 480, 23 (2000); Eur. Phys. J. C 18, 283 (2000).

[35] A. Bottino, F. Donato, N. Fornengo, and S. Scopel, Phys. Lett. B 423, 109 (1998); Phys. Rev. D 62, 056006 (2000); 63, 125003 (2001).

[36] R.H. Helm, Phys. Rev. 104, 1466 (1956).

[37] A. Bottino, F. Donato, G. Mignola, S. Scopel, P. Belli, and A. Incicchitti, Phys. Lett. B 402, 113 (1997).

[38] P. Belli, R. Cerulli, N. Fornengo, and S. Scopel, Phys. Rev. D 66, 043503 (2002). 